\title{
Thank you to Virology Journal's peer reviewers in 2014
}

Linfa Wang ${ }^{1,2}$

\section{Contributing reviewers}

The editors of Virology Journal would like to thank all our reviewers who have contributed to the journal in Volume 11 (2014). The success of any scientific journal depends on an effective and strict peer review process and Virology Journal could not operate without your contribution. We are grateful to the large number of reviewers, who have done a great job in not only lifting the quality of the journal's scientific peer reviewing process, but also helped us to reach a median time to first decision of 39 days.

We look forward to your continuous support of Virology Journal either as an invited reviewer or a contributing author in the years to come.

Maria Aamelfot

Norway

Jorge Abad

USA

Ahmed Abd El Wahed

Germany

Jônatas Abrahão

Brazil

Akio Adachi
Japan

Claudio Afonso

USA

Stephen Ahern

USA

Jameleddine Aissa Larousse

Tunisia

Mohammed Al-Ahdal

Saudi Arabia

Annika Allard

Sweden
Andrew Allison

USA

Sidney Altman

USA

Barry Alto

USA

Alberto A Amarilla

Brazil

Jaime Henrique Amorim

Brazil

Maria Joao Amorim

Portugal

Nisalak Ananda

Thailand

Victor H Aquino

Brazil

Juan Arbiza

Uruguay

Carlos Arias

Mexico
Jiro Arikawa

Japan

Minetaro Arita

Japan

Luciana Arruda

Brazil

Jose T. Ascencio-Ibanez

USA

Antonio Ascione

Italy

Sassan Asgari

Australia

Claire Atkinson

UK

Eduardo Azziz-Baumgartner

USA

Leen Baert

Switzerland

Olfa Bahri

Tunisia

Correspondence: Linfa.Wang@csiro.au

${ }^{1}$ CSIRO Animal Food and Health Sciences, Australian Animal Health

Laboratory, Geelong, VIC 3220, Australia

${ }^{2}$ Program in Emerging Infectious Diseases, Duke-NUS Graduate Medical

School, Singapore 169857, Singapore

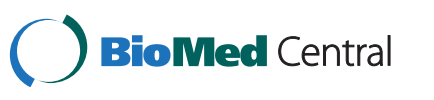

(c) 2015 Wang; licensee BioMed Central. This is an Open Access article distributed under the terms of the Creative Commons Attribution License (http://creativecommons.org/licenses/by/4.0), which permits unrestricted use, distribution, and reproduction in any medium, provided the original work is properly credited. The Creative Commons Public Domain Dedication waiver (http://creativecommons.org/publicdomain/zero/1.0/) applies to the data made available in this article, unless otherwise stated. 


\begin{tabular}{|c|c|c|}
\hline $\begin{array}{l}\text { Susan Baker } \\
\text { USA }\end{array}$ & $\begin{array}{l}\text { Bishnupriya Bhattacharya } \\
\text { UK }\end{array}$ & $\begin{array}{l}\text { Alexander Bukreyev } \\
\text { USA }\end{array}$ \\
\hline $\begin{array}{l}\text { Judith Ball } \\
\text { USA }\end{array}$ & $\begin{array}{l}\text { Huijie Bian } \\
\text { China }\end{array}$ & $\begin{array}{l}\text { Cara Burns } \\
\text { USA }\end{array}$ \\
\hline $\begin{array}{l}\text { Subrata Banerjee } \\
\text { India }\end{array}$ & $\begin{array}{l}\text { Marina Bidin } \\
\text { Croatia }\end{array}$ & $\begin{array}{l}\text { Michael Burwinkel } \\
\text { Germany }\end{array}$ \\
\hline $\begin{array}{l}\text { Celia Barardi } \\
\text { Brazil }\end{array}$ & $\begin{array}{l}\text { John Blaho } \\
\text { USA }\end{array}$ & $\begin{array}{l}\text { Pierre Busson } \\
\text { France }\end{array}$ \\
\hline $\begin{array}{l}\text { Edel Barbosa-Stancioli } \\
\text { Brazil }\end{array}$ & $\begin{array}{l}\text { Sandra Blome } \\
\text { Germany }\end{array}$ & $\begin{array}{l}\text { James Campbell } \\
\text { Canada }\end{array}$ \\
\hline $\begin{array}{l}\text { Michael Baron } \\
\text { UK }\end{array}$ & $\begin{array}{l}\text { Hubert Blum } \\
\text { Germany }\end{array}$ & $\begin{array}{l}\text { Patricia Cane } \\
\text { UK }\end{array}$ \\
\hline $\begin{array}{l}\text { John Barr } \\
\text { UK }\end{array}$ & $\begin{array}{l}\text { Karl Boehme } \\
\text { USA }\end{array}$ & $\begin{array}{l}\text { Chunxia Cao } \\
\text { USA }\end{array}$ \\
\hline $\begin{array}{l}\text { Erik Barton } \\
\text { USA }\end{array}$ & $\begin{array}{l}\text { Andrew Bohm } \\
\text { USA }\end{array}$ & $\begin{array}{l}\text { Ya Cao } \\
\text { China }\end{array}$ \\
\hline $\begin{array}{l}\text { Chris Basler } \\
\text { USA }\end{array}$ & $\begin{array}{l}\text { Myrna Bonaldo } \\
\text { Brazil }\end{array}$ & $\begin{array}{l}\text { Antonella Caputo } \\
\text { Italy }\end{array}$ \\
\hline $\begin{array}{l}\text { Lorena Bavia } \\
\text { Brazil }\end{array}$ & $\begin{array}{l}\text { Cláudio Bonjardim } \\
\text { Brazil }\end{array}$ & $\begin{array}{l}\text { Tereza Cardoso } \\
\text { Brazil }\end{array}$ \\
\hline $\begin{array}{l}\text { Bhupinder Bawa } \\
\text { USA }\end{array}$ & $\begin{array}{l}\text { Alessandra Borges } \\
\text { Brazil }\end{array}$ & $\begin{array}{l}\text { Faizal Careem } \\
\text { Canada }\end{array}$ \\
\hline $\begin{array}{l}\text { Eduardo Bejarano } \\
\text { Spain }\end{array}$ & $\begin{array}{l}\text { Guglielmo Borgia } \\
\text { Italy }\end{array}$ & $\begin{array}{l}\text { Claudio Caruso } \\
\text { Italy }\end{array}$ \\
\hline $\begin{array}{l}\text { Brendan Bell } \\
\text { Canada }\end{array}$ & $\begin{array}{l}\text { Silvia Boscardin } \\
\text { Brazil }\end{array}$ & $\begin{array}{l}\text { Elena Catelli } \\
\text { Italy }\end{array}$ \\
\hline $\begin{array}{l}\text { Dirk Bellstedt } \\
\text { South Africa }\end{array}$ & $\begin{array}{l}\text { Crystal Boudreaux } \\
\text { USA }\end{array}$ & $\begin{array}{l}\text { Luciano Cavalcanti } \\
\text { Brazil }\end{array}$ \\
\hline $\begin{array}{l}\text { Jessica Belser } \\
\text { USA }\end{array}$ & $\begin{array}{l}\text { Andrew Bowman } \\
\text { USA }\end{array}$ & $\begin{array}{l}\text { Mattia Cecchinato } \\
\text { Italy }\end{array}$ \\
\hline $\begin{array}{l}\text { Dennis Bente } \\
\text { USA }\end{array}$ & $\begin{array}{l}\text { Paulo Brandao } \\
\text { Brazil }\end{array}$ & $\begin{array}{l}\text { Remi Charrel } \\
\text { France }\end{array}$ \\
\hline $\begin{array}{l}\text { Vincent Beringue } \\
\text { France }\end{array}$ & $\begin{array}{l}\text { Laurence Briant } \\
\text { France }\end{array}$ & $\begin{array}{l}\text { Animesh Chatterjee } \\
\text { USA }\end{array}$ \\
\hline $\begin{array}{l}\text { Ben Berkhout } \\
\text { Netherlands }\end{array}$ & $\begin{array}{l}\text { David Brighty } \\
\text { UK }\end{array}$ & $\begin{array}{l}\text { Yahia Chebloune } \\
\text { France }\end{array}$ \\
\hline $\begin{array}{l}\text { Jesus F Bermejo-Martin } \\
\text { Spain }\end{array}$ & $\begin{array}{l}\text { Christopher Broder } \\
\text { USA }\end{array}$ & $\begin{array}{l}\text { Gerald Kimani Chege } \\
\text { South Africa }\end{array}$ \\
\hline $\begin{array}{l}\text { Charles Berry } \\
\text { USA }\end{array}$ & $\begin{array}{l}\text { Ivan Brukner } \\
\text { Canada }\end{array}$ & $\begin{array}{l}\text { En-Qiang Chen } \\
\text { China }\end{array}$ \\
\hline $\begin{array}{l}\text { Mael Bessaud } \\
\text { France }\end{array}$ & $\begin{array}{l}\text { Hubert Buczkowski } \\
\text { UK }\end{array}$ & $\begin{array}{l}\text { Jen-Yang Chen } \\
\text { Taiwan }\end{array}$ \\
\hline $\begin{array}{l}\text { Ramya Bhatia } \\
\text { UK }\end{array}$ & $\begin{array}{l}\text { Gertrude Buehring } \\
\text { USA }\end{array}$ & $\begin{array}{l}\text { Jianfei Chen } \\
\text { China }\end{array}$ \\
\hline
\end{tabular}


Ji-Ming Chen

China

Limin Chen

Canada

Quanjiao Chen

China

Tong-Xin Chen

China

Xinwen Chen

China

Zhongbin Chen

China

Anchun Cheng

China

Tong Cheng

China

Zhaobang Cheng

China

Sarah Cherian

India

Bor-Luen Chiang

Taiwan

Chiara Chiapponi

Italy

Mohamad Chikh Ali

USA

V Gregory Chinchar

USA

Hui-Ling Chiou

Taiwan

Christopher Chiu

UK

Susan Chiu

Hong Kong

Kyoung-Oh Cho

Korea, South

Bum Soon Choi

Korea, South

Jin Huk Choi

USA

Kyoung-Seong Choi

Korea, South

Pele Chong

Taiwan
Yen-Hung Chow

Taiwan

Wan-Long Chuang

Taiwan

Pauline Chugh

USA

Konstantin Chumakov

USA

Ju-Young Chung

Korea, South

Massimo Ciccozzi

Italy

Christine Elisabeth Clavel

France

Carla Coffin

Canada

Philippe Colson

France

Sarah Connolly

USA

Samuel Cordey

Switzerland

Anthony Corfield

UK

Alexandre Costa

Brazil

Andre Costa-da-Silva

Brazil

Maria Isabel Costafreda USA

Veronica Costantini

USA

Therese Couderc

Gabon

Brenda Coutts

Australia

Manuel Crespo

Spain

Helen Crooke

UK

Robert Cross

USA

Edson da Silva

Brazil
Ajmal Dalwai

Kuwait

Armando Damiani

Germany

Elsa Damonte

Argentina

Andrew Davidson

UK

Mohamed Ali Daw

Libya

Craig Day

USA

Damián F. de Andrés

Spain

Luana de Borba

Argentina

Juan Carlos de la Torre

USA

Carlos de Noronha

USA

Robert de Vries

USA

Emmie de Wit

USA

Nicola Decaro

Italy

Rosa del Angel

Mexico

Jose Del Campo

Spain

Adriana Delfraro

Uruguay

Gustavo Delhon

USA

Eric Delwart

USA

Joachim Denner

Germany

Moutaz Derbala

Qatar

Tararaj Dharakul

Thailand

Adarsh Dharan

USA 
Barbara Di Martino

Italy

Detlef E. Dietrich

Germany

Paul Digard

UK

Chan Ding

China

Ulf Dittmer

Germany

Maurizio Divizia

Italy

Stephen Dollery

USA

Irini Doytchinova

Bulgaria

Jan Felix Drexler

Germany

Edward Dubovi

USA

Robert Duda

USA

Stephen Dunham

UK

Rebecca Dutch

USA

Andrew Easton

UK

Jose Echevarria

Spain

Maryna Eichelberger

USA

Anna Eis-Huebinger

Germany

Manal El-Sayed

Egypt

Othmar Engelhardt

UK

Luis Enjuanes

Spain

Jose M Escribano

Spain

Pedro Esteves

Portugal
Jennifer Evans

UK

James Evermann

USA

Catherine Ewen

USA

Shufang Fan

USA

Minggang Fang

USA

A. Hossain Farid

Canada

Francesco Feletti

Italy

Wenhai Feng

China

Ricardo Flores

Spain

Robert Flower

Australia

Caroline Fossum

Sweden

Jeffrey Foster

USA

Sandra Frabasile

Uruguay

Rennos Fragkoudis

UK

Silvia Franceschi

France

Conrad Freuling

Germany

Jean-Pierre Frossard

UK

Cristina Fuentes Pardo

Spain

Masashi Fukayama

Japan

Monica Galiano

UK

Donato Gallitelli

Italy

Feng Gao

China
Feng Gao

USA

Antonio Garmendia

USA

Lana Garmire

USA

Silvana Gaudieri

Australia

Ritu Gaur

India

Brian Geiss

USA

Elke Genersch

Germany

Abdeljelil Ghram

Tunisia

Adrian Gibbs

Australia

Ivone Giffard-Mena

Mexico

Jason Gill

USA

Andre Gomes

Brazil

Jordi Gomez

Spain

Esperanza Gomez-Lucia

Spain

Jean-Paul Gonzalez

USA

Radhika Gopal

USA

Hideo Goto

Japan

Maruthi M N Gowda

UK

Urs Greber

Switzerland

Giorgio Gribaudo

Italy

Anthony Griffiths

USA

Charles Grose

USA 


\begin{tabular}{|c|c|c|}
\hline $\begin{array}{l}\text { Christian Grund } \\
\text { Germany }\end{array}$ & $\begin{array}{l}\text { Graham Hatfull } \\
\text { USA }\end{array}$ & $\begin{array}{l}\text { Ivan Hung } \\
\text { Hong Kong }\end{array}$ \\
\hline $\begin{array}{l}\text { Linlin Gu } \\
\text { USA }\end{array}$ & $\begin{array}{l}\text { Daisuke Hayasaka } \\
\text { Japan }\end{array}$ & $\begin{array}{l}\text { Elizabeth Hunsperger } \\
\text { Puerto Rico }\end{array}$ \\
\hline $\begin{array}{l}\text { Rodrigo Guabiraba } \\
\text { France }\end{array}$ & $\begin{array}{l}\text { Qigai He } \\
\text { China }\end{array}$ & $\begin{array}{l}\text { Dae Young Hur } \\
\text { Korea, South }\end{array}$ \\
\hline $\begin{array}{l}\text { Larisa Gubareva } \\
\text { USA }\end{array}$ & $\begin{array}{l}\text { Isabelle Heard } \\
\text { France }\end{array}$ & $\begin{array}{l}\text { Aeron Hurt } \\
\text { Australia }\end{array}$ \\
\hline $\begin{array}{l}\text { Maria Isabel Guedes } \\
\text { Brazil }\end{array}$ & $\begin{array}{l}\text { Alireza Heidari } \\
\text { Italy }\end{array}$ & $\begin{array}{l}\text { Gisela Hussey } \\
\text { USA }\end{array}$ \\
\hline $\begin{array}{l}\text { Hailong Guo } \\
\text { USA }\end{array}$ & $\begin{array}{l}\text { Terho Heikkinen } \\
\text { Finland }\end{array}$ & $\begin{array}{l}\text { Edward Hutchinson } \\
\text { UK }\end{array}$ \\
\hline $\begin{array}{l}\text { Haitao Guo } \\
\text { USA }\end{array}$ & $\begin{array}{l}\text { Regine Heilbronn } \\
\text { Germany }\end{array}$ & $\begin{array}{l}\text { Ali Idris } \\
\text { USA }\end{array}$ \\
\hline $\begin{array}{l}\text { Xiaofeng Guo } \\
\text { China }\end{array}$ & $\begin{array}{l}\text { Andrew Henderson } \\
\text { USA }\end{array}$ & $\begin{array}{l}\text { Kyung-Soo Inn } \\
\text { Korea, South }\end{array}$ \\
\hline $\begin{array}{l}\text { Ahmet Gurakar } \\
\text { USA }\end{array}$ & $\begin{array}{l}\text { Lara Herrero } \\
\text { Australia }\end{array}$ & $\begin{array}{l}\text { Will Irving } \\
\text { UK }\end{array}$ \\
\hline $\begin{array}{l}\text { Jon Gutierrez } \\
\text { Slovenia }\end{array}$ & $\begin{array}{l}\text { Guy Hervé } \\
\text { France }\end{array}$ & $\begin{array}{l}\text { Charles Isaacs } \\
\text { USA }\end{array}$ \\
\hline $\begin{array}{l}\text { Clifford Guy } \\
\text { USA }\end{array}$ & $\begin{array}{l}\text { Toshiki Himeda } \\
\text { Japan }\end{array}$ & $\begin{array}{l}\text { Masashi Iwanaga } \\
\text { Japan }\end{array}$ \\
\hline $\begin{array}{l}\text { James Guy } \\
\text { USA }\end{array}$ & $\begin{array}{l}\text { Jung Joo Hong } \\
\text { USA }\end{array}$ & $\begin{array}{l}\text { Alan Jackson } \\
\text { Canada }\end{array}$ \\
\hline $\begin{array}{l}\text { Bart Haagmans } \\
\text { Netherlands }\end{array}$ & $\begin{array}{l}\text { Daniel Horton } \\
\text { UK }\end{array}$ & $\begin{array}{l}\text { Richard Jackson } \\
\text { UK }\end{array}$ \\
\hline $\begin{array}{l}\text { Hans Haecker } \\
\text { USA }\end{array}$ & $\begin{array}{l}\text { Katja Hoschler } \\
\text { UK }\end{array}$ & $\begin{array}{l}\text { Daral Jackwood } \\
\text { USA }\end{array}$ \\
\hline $\begin{array}{l}\text { Roy Hall } \\
\text { Australia }\end{array}$ & $\begin{array}{l}\text { Margaret Hosie } \\
\text { UK }\end{array}$ & $\begin{array}{l}\text { Lisa Jameson } \\
\text { UK }\end{array}$ \\
\hline $\begin{array}{l}\text { Xinbing Han } \\
\text { USA }\end{array}$ & $\begin{array}{l}\text { Hak Hotta } \\
\text { Japan }\end{array}$ & $\begin{array}{l}\text { Shijin Jiang } \\
\text { China }\end{array}$ \\
\hline $\begin{array}{l}\text { Ken-Ichi Hanaki } \\
\text { Japan }\end{array}$ & $\begin{array}{l}\text { Weihong Hou } \\
\text { USA }\end{array}$ & $\begin{array}{l}\text { Emma Job } \\
\text { Australia }\end{array}$ \\
\hline $\begin{array}{l}\text { Tomas Hanke } \\
\text { UK }\end{array}$ & $\begin{array}{l}\text { Cheng Huang } \\
\text { USA }\end{array}$ & $\begin{array}{l}\text { Reimar Johne } \\
\text { Germany }\end{array}$ \\
\hline $\begin{array}{l}\text { Kathryn Hanley } \\
\text { USA }\end{array}$ & $\begin{array}{l}\text { Li-Rung Huang } \\
\text { Taiwan }\end{array}$ & $\begin{array}{l}\text { Nicholas Johnson } \\
\text { UK }\end{array}$ \\
\hline $\begin{array}{l}\text { Chetan Hans } \\
\text { USA }\end{array}$ & $\begin{array}{l}\text { Yaowei Huang } \\
\text { USA }\end{array}$ & $\begin{array}{l}\text { Ho Won Jung } \\
\text { Korea, South }\end{array}$ \\
\hline $\begin{array}{l}\text { Gordon Harkins } \\
\text { South Africa }\end{array}$ & $\begin{array}{l}\text { Yu Huang } \\
\text { China }\end{array}$ & $\begin{array}{l}\text { Sandra Junglen } \\
\text { Germany }\end{array}$ \\
\hline $\begin{array}{l}\text { Heli Harvala } \\
\text { UK }\end{array}$ & $\begin{array}{l}\text { Christine Hughes } \\
\text { Canada }\end{array}$ & $\begin{array}{l}\text { Adrian Kajon } \\
\text { USA }\end{array}$ \\
\hline
\end{tabular}




\begin{tabular}{|c|c|c|}
\hline Robert Kalejta & Boris Klempa & Karen Laurie \\
\hline USA & Slovakia & Australia \\
\hline Tatsuo Kanda & Maja Kodani & James Lawson \\
\hline USA & USA & Australia \\
\hline Hisatoshi Kaneko & Jeffrey Koehler & Jen Layden \\
\hline Japan & USA & USA \\
\hline Amit Kapoor & Andrea Koishi & Thomas Layden \\
\hline USA & Brazil & USA \\
\hline Hiroaki Kariwa & Eva Krause & Chuan-Mo Lee \\
\hline Japan & Germany & Taiwan \\
\hline Dietmar Katinger & Astrid Krmpotic & Joong Bok Lee \\
\hline Austria & Croatia & Korea, South \\
\hline Benedikt Kaufer & Andrew Kropinski & David Lefebvre \\
\hline Germany & Canada & Belgium \\
\hline Ghazi Kayali & Peter Krug & Elliot Lefkowitz \\
\hline USA & USA & USA \\
\hline Alyson Kelvin & Detlev H Kruger & Guy Lemay \\
\hline Canada & Germany & Canada \\
\hline Pattara Khamrin & Claude Krummenacher & Niels A.W. Lemmermann \\
\hline Thailand & USA & Germany \\
\hline Mohsin Khan & Diogo Kuczera & Min Levine \\
\hline USA & Brazil & USA \\
\hline Madhu Khanna & Ajit Kumar & Avram Levy \\
\hline India & USA & Australia \\
\hline Sunil Khattar & Sachin Kumar & Jay Levy \\
\hline USA & India & USA \\
\hline Alexander Khromykh & Stefan Kunz & Chengjun Li \\
\hline Australia & Switzerland & China \\
\hline Hyun Soo Kim & Lise Kirstine Kvisgaard & Lanjuan Li \\
\hline Korea, South & Denmark & China \\
\hline Seong-Jun Kim & Giuseppina La Rosa & Linlin Li \\
\hline USA & Italy & USA \\
\hline Shin-Hee Kim & Ole Lagatie & Mei-Ling Li \\
\hline USA & Belgium & USA \\
\hline David Kimberlin & Alexander Lai & Shifang Li \\
\hline USA & Hong Kong & China \\
\hline Lóránt Király & Yinghua Lan & Min Liao \\
\hline Hungary & China & China \\
\hline Peter Kirkland & Stephen E. Lapinsky & Daniel Libraty \\
\hline Australia & Canada & USA \\
\hline Irina Kiseleva & Tomasz Laskus & Magnus Lindh \\
\hline Russia & Poland & Sweden \\
\hline Leera Kittigul & Susanna Lau & Fenyong Liu \\
\hline Thailand & Hong Kong & USA \\
\hline
\end{tabular}


Jinhua Liu

China

Qinfang Liu

USA

Francisco Lobo

Brazil

Martin Loessner

Switzerland

Huaguang Lu

USA

Michaela Lucas

Australia

Juan Ludert

Mexico

Catherine Luke

USA

Shuhong Luo

China

Blanca Lupiani

USA

Hinh Ly

USA

Samantha Lycett

UK

Daoxin Ma

China

Guanggang $\mathrm{Ma}$

China

Liqing Ma

China

Yijie Ma

USA

Dominiek Maes

Belgium

Padmanabhan Mahadevan

USA

Eugene Major

USA

Shinji Makino

USA

Carlos Maluquer de Motes

UK

Nicholas Maness

USA
Balaji Manicassamy

USA

Anna Liza Manlutac

USA

Spilios Manolakopoulos

Greece

Atika Mansoor

Pakistan

María Rosa Marano

Argentina

Sylvie Marché

Belgium

Denise Marston

UK

Contigiani Marta

Argentina

Darren Martin

South Africa

Miguel A Martin-acebes

Spain

Isidoro Martinez

Spain

Miguel Angel Martinez

Spain

Encarna Martinez Salas

Spain

Javier Martinez-Picado

Spain

Luis Martinez-Sobrido

USA

Regina Martins

Brazil

Vilma Martins

Brazil

William Mason

USA

Enric Mateu

Spain

Masanori Matsui

Japan

Yumiko Matsuoka

USA

Shohei Matsuura

Japan
Giada Mattiuzzo

UK

Leena Maunula

Finland

Sebastian Maurer-Stroh

Singapore

Axel Mauroy

Belgium

Budhaditya Mazumdar

USA

Urszula Mazurek

Poland

Julie McAuley

Australia

Harris McFerrin

USA

Gerald McInerney

Sweden

John McKillen

UK

Gary McLean

UK

José A. Melero

Spain

Baozhong Meng

Canada

Janet Mertz

USA

Andrew Mesecar

USA

Francois Meurens

Canada

Florencia Meyer

Uruguay

Andreas Meyerhans

Spain

Scott Michael

USA

Thomas Michalak

Canada

Luiz Miletti

Brazil

David Milich

USA 
Patti Miller

USA

Hyeyoung Min

Korea, South

Chad Mire

USA

Nischay Mishra

USA

Tadayoshi Mitsuhashi

Japan

Katsumi Mizuta

Japan

Susanne Modrow

Germany

Ugo Moens

Norway

Ka Pun Mok

Hong Kong

Eamonn Molloy

Ireland

Isabella Monne

Italy

Ronald Montelaro

USA

Emanuele Montomoli

Italy

Cary Moody

USA

Christiane Moog

France

Martin Moore

USA

Tsafrir Mor

USA

Luiz Tadeu Moraes Figueiredo

Brazil

Norio Mori

Japan

Enrique Moriones

Spain

Rhoda Morrow

USA

Eric Mossel

USA
Bruno Mota

Brazil

Nissin Moussatche

USA

Sunil Mukherjee

India

Marcel Müller

Germany

Patricia Mulrooney-Cousins

Canada

Muhammad Munir

UK

Yoshiki Murakami

Japan

William Murphy

USA

Jonah Musa

Nigeria

Yael Mutsafi

Israel

Greg Mutze

Australia

Eva Nagy

Canada

Keisuke Nakagawa

Japan

Scott Napper

Canada

Krishna Narayanan

USA

Farooq Nasar

USA

Beatriz Navarro

Italy

Jesus Navas-Castillo

Spain

Somanna Naveen

USA

Clive Naylor

UK

Jean Ndjomou

USA

Daniel Nelson

USA
Laura Newcomb

USA

Johan Neyts

Belgium

Terry Fei Fan Ng

USA

Tsai Nga Wing Polly

Hong Kong

John Nicholls

Hong Kong

Zuoming Nie

China

Veljko Nikolin

Germany

Yorihiro Nishimura

Japan

Andreas Nitsche

Germany

Juliana Nogueira

Brazil

Helene Norder

Sweden

Marcio Nunes

Brazil

Mark O'Dea

Australia

Leslie Ogorzaly

Luxembourg

Aileen O'Hearn

USA

Seii Ohka

Japan

Judith Olejnik

USA

Gene Olinger

USA

Hugo Oliveira

Portugal

John Daniel O'Neil

UK

Eleonore Ostermann

Germany

Masao Ota

Japan 


\begin{tabular}{|c|c|c|}
\hline David Ott & Jean-Pierre Perreault & Krzysztof Pyrc \\
\hline USA & Canada & Poland \\
\hline Qishui Ou & Martin Pfeffer & Zhikang Qian \\
\hline China & Germany & China \\
\hline Paula Padula & Sébastien Pfeffer & Chao Qiu \\
\hline Argentina & France & China \\
\hline Anandan Paldurai & Tram Pham & Jianming Qiu \\
\hline USA & Canada & USA \\
\hline Peter Palese & Tung Phan & Josep Quer \\
\hline USA & USA & Spain \\
\hline Ann Palmenberg & Nittaya Phanuphak & Shafquat Rafiq \\
\hline USA & Thailand & UK \\
\hline Ana Luiza Pamplona Mosimann & Jaime Pignatelli & Jaya Rajaiya \\
\hline Brazil & Spain & USA \\
\hline Mary Pantin-Jackwood & Gorben Pijlman & Yogendra Rajawat \\
\hline USA & Netherlands & USA \\
\hline Hanu Pappu & Benjamin Pinsky & Huiying Rao \\
\hline USA & USA & China \\
\hline Mark Parcells & Aguinaldo Pinto & Adam Rash \\
\hline USA & Brazil & UK \\
\hline Satya Parida & Rosa Maria Pintó & Thomas Bruun Rasmussen \\
\hline UK & Spain & Denmark \\
\hline Joanna Parish & Antonio Piralla & Jorgen Ravoet \\
\hline UK & Italy & Belgium \\
\hline Bongkyun Park & Mauro Pistello & William Rawlinson \\
\hline Korea, South & Italy & Australia \\
\hline Rebecca Parr & Justin Pita & Simon Rayner \\
\hline USA & USA & China \\
\hline Colin Parrish & Stefan Pöhlmann & Cath Rees \\
\hline USA & Germany & UK \\
\hline Kunj Pathak & Welkin Pope & Ilona Reimann \\
\hline USA & USA & Germany \\
\hline John Patton & Hamid Pourianfar & Chantal Reusken \\
\hline USA & Iran & Netherlands \\
\hline Ben Peeters & Ali Pouryasin & Mathilde Richard \\
\hline Netherlands & Iran & Netherlands \\
\hline Daxin Peng & Ramesh Prabhu & Elizabeth Rieder \\
\hline China & USA & USA \\
\hline Yihong Peng & Martina Prelog & Daniel Ripoll \\
\hline China & Germany & USA \\
\hline Luciane Maria Perazzolo & Joseph Prescott & Guillermo Risatti \\
\hline Brazil & USA & USA \\
\hline Carlos Pereira & Cinta Prieto & Pierre Rivailler \\
\hline Brazil & Spain & USA \\
\hline
\end{tabular}


Rafael F. Rivera-Bustamante

Mexico

Steve Rockman

Australia

Barry Rockx

USA

Julio Rodriguez

Australia

Pierre Roques

France

Daniela Rosa

Brazil

German Rosas-Acosta

USA

Anca Roseanu

Romania

Helene Rosenberg

USA

Susan Ross

USA

Michael G Rossmann

USA

Raymond Rowland

USA

Dora Ruchansky

Uruguay

Franco Maria Ruggeri

Italy

Nicolas Ruggli

Switzerland

Alvaro Ruiz

Chile

Jonathan Runstadler

USA

Wi-Sun Ryu

Korea, South

Armin Saalmueller

Austria

Ali Sabahi

USA

Ivan Sadowski

Canada

Mahmut Safak

USA
Dipongkor Saha

USA

Nitin Saksena

Australia

Francisco Javier Salguero

UK

Teruo Sano

Japan

Flavia Santos

Brazil

Daniel Santos Mansur

Brazil

Yoshikuni Sato

Japan

Benjamin Satterfield

USA

Andreas Sauerbrei

Germany

Carita Savolainen-Kopra

Finland

Hirofumi Sawa

Japan

Paula Schaffer

USA

Luis Schang

Canada

Horst Schirrmeier

Germany

Jeffrey Schneider

USA

Juergen Schneider-Schaulies

Germany

Amy Schuh

USA

Anna Charlotte Schultz

Denmark

Martin Schwemmle

Germany

Michel Segondy

France

Bert Semler

USA

Noemi Sevilla

Spain
Norazizah Shafee

Malaysia

Ruiping She

China

Noula Shembade

USA

Huigang Shen

China

Tao Shen

China

Jishu Shi

USA

Yusuke Shimakawa

Japan

Maya Shmulevitz

Canada

Guilherme Silveira

Brazil

Rodrigo Silvestre

Brazil

Valerie Sim

Canada

Peter Simmonds

UK

David Skibinski

Singapore

Mikael Skurnik

Finland

Anny Slama-Schwok

France

Marek Smieja

Canada

Teemu Smura

Finland

Chantal Snoeck

Luxembourg

Muhammad Sohail

USA

Chang-Seon Song

Korea, South

Daniele Souza

Brazil

Doris Souza

Brazil 
Erica Spackman

USA

Tim Sparer

USA

Fernando Spilki

Brazil

Burke Squires

USA

Peter Staeheli

Germany

Thomas Stamminger

Germany

Martin Steinau

USA

Bettie Steinberg

USA

Stephen Stray

USA

Rebecca Strong

UK

Daisy Strottmann

Brazil

Elankumaran Subbiah

USA

Christopher Sullivan

USA

Jack M. Sullivan

USA

Artur Summerfield

Switzerland

Rebecca Sumner

UK

Petri Susi

Finland

Leonardo Susta

USA

Gerd Sutter

Germany

Troy Sutton

USA

Gulam Syed

USA

Yoichi Takahagi

Japan

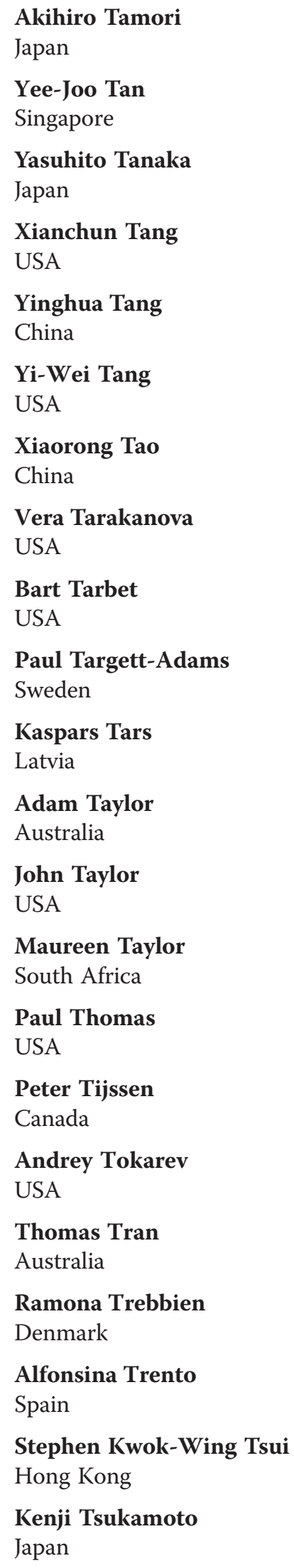

Akihiro Tamori

Yee-Joo Tan

Singapore

Yasuhito Tanaka

Xianchun Tang

China

Yi-Wei Tang

Xiaorong Tao

Vera Tarakanova

USA

Bart Tarbet

Paul Targett-Adams

Kaspars Tars

Adam Taylor

Astralia

John Taylor

Maureen Taylor

South Africa

Paul Thomas

Peter Tijssen

Andrey Tokarev

USA

Australia

Ramona Trebbien

Alfonsina Trento

Stephen Kwok-Wing Tsui

ong Kong

Japan
Changchun $\mathrm{Tu}$

China

Edan Tulman

USA

Tobias Tuthill

UK

Sukathida Ubol

Thailand

Takeji Umemura

Japan

Wim Van der Poel

Netherlands

James Van Etten

USA

Frank van Kuppeveld

Netherlands

Herve Vanderschuren

Switzerland

Arvind Varsani

New Zealand

Francisco Veas

France

Michael Veit

Germany

Georges Verjans

Netherlands

Newton Medeiros Vidal

Brazil

Alexandre Vieira Machado

Brazil

Dhanasekaran Vijaykrishna

Singapore

Stefan Vilcek

Slovakia

Heiner von Buttlar

Germany

Ann Vossen

Netherlands

Abdul Waheed

USA

Peter Walker

Australia

Per Wallgren

Sweden 


\begin{tabular}{|c|c|c|}
\hline $\begin{array}{l}\text { Hongquan Wan } \\
\text { USA }\end{array}$ & $\begin{array}{l}\text { William Wilson } \\
\text { USA }\end{array}$ & $\begin{array}{l}\text { Yoshihiko Yano } \\
\text { Japan }\end{array}$ \\
\hline $\begin{array}{l}\text { Xiu-Feng (Henry) Wan } \\
\text { USA }\end{array}$ & $\begin{array}{l}\text { Helen Wise } \\
\text { UK }\end{array}$ & $\begin{array}{l}\text { Chau-Ting Yeh } \\
\text { Taiwan }\end{array}$ \\
\hline $\begin{array}{l}\text { Jianwei Wang } \\
\text { China }\end{array}$ & $\begin{array}{l}\text { Roland Wolkowicz } \\
\text { USA }\end{array}$ & $\begin{array}{l}\text { Man Lung Yeung } \\
\text { Hong Kong }\end{array}$ \\
\hline $\begin{array}{l}\text { Robert YL Wang } \\
\text { Taiwan }\end{array}$ & $\begin{array}{l}\text { Sek-Man Wong } \\
\text { Singapore }\end{array}$ & $\begin{array}{l}\text { Shounan Yi } \\
\text { Australia }\end{array}$ \\
\hline $\begin{array}{l}\text { Tian Wang } \\
\text { USA }\end{array}$ & $\begin{array}{l}\text { Gary Wormser } \\
\text { USA }\end{array}$ & $\begin{array}{l}\text { Lawrence Young } \\
\text { UK }\end{array}$ \\
\hline $\begin{array}{l}\text { Xiaomei Wang } \\
\text { China }\end{array}$ & $\begin{array}{l}\text { Grzegorz Jan Wozniakowski } \\
\text { Poland }\end{array}$ & $\begin{array}{l}\text { Youxiang Diao } \\
\text { China }\end{array}$ \\
\hline $\begin{array}{l}\text { Wanzhe Yuan } \\
\text { China }\end{array}$ & $\begin{array}{l}\text { Changde Wu } \\
\text { China }\end{array}$ & $\begin{array}{l}\text { Di Yu } \\
\text { Australia }\end{array}$ \\
\hline $\begin{array}{l}\text { Gulam Waris } \\
\text { USA }\end{array}$ & $\begin{array}{l}\text { Ho-sheng Wu } \\
\text { Taiwan }\end{array}$ & $\begin{array}{l}\text { Li Yu } \\
\text { China }\end{array}$ \\
\hline $\begin{array}{l}\text { Peter Wark } \\
\text { Australia }\end{array}$ & $\begin{array}{l}\text { Dongbo Wu } \\
\text { China }\end{array}$ & $\begin{array}{l}\text { Ming-Lung Yu } \\
\text { Taiwan }\end{array}$ \\
\hline $\begin{array}{l}\text { Simone Warner } \\
\text { Australia }\end{array}$ & $\begin{array}{l}\text { Xiaofeng Wu } \\
\text { China }\end{array}$ & $\begin{array}{l}\text { Qingzhong Yu } \\
\text { USA }\end{array}$ \\
\hline $\begin{array}{l}\text { Shumpei Watanabe } \\
\text { Japan }\end{array}$ & $\begin{array}{l}\text { Zhiyong Xi } \\
\text { USA }\end{array}$ & $\begin{array}{l}\text { Hang Yuan } \\
\text { USA }\end{array}$ \\
\hline $\begin{array}{l}\text { Koichi Watashi } \\
\text { Japan }\end{array}$ & $\begin{array}{l}\text { Shi-Hua Xiang } \\
\text { USA }\end{array}$ & $\begin{array}{l}\text { Yuchen Fan } \\
\text { China }\end{array}$ \\
\hline $\begin{array}{l}\text { Nadia Wauquier } \\
\text { France }\end{array}$ & $\begin{array}{l}\text { Shu-Yuan Xiao } \\
\text { USA }\end{array}$ & $\begin{array}{l}\text { Zeng Yue } \\
\text { China }\end{array}$ \\
\hline $\begin{array}{l}\text { Eefke Weesendorp } \\
\text { Netherlands }\end{array}$ & $\begin{array}{l}\text { Peng Xie } \\
\text { China }\end{array}$ & $\begin{array}{l}\text { Yuehuan Liu } \\
\text { China }\end{array}$ \\
\hline $\begin{array}{l}\text { Lai Wei } \\
\text { China }\end{array}$ & $\begin{array}{l}\text { Youhua Xie } \\
\text { China }\end{array}$ & $\begin{array}{l}\text { Kwok Yung Yuen } \\
\text { Hong Kong }\end{array}$ \\
\hline $\begin{array}{l}\text { Manfred Weidmann } \\
\text { UK }\end{array}$ & $\begin{array}{l}\text { Xiyan Xu } \\
\text { USA }\end{array}$ & $\begin{array}{l}\text { Nadezhda Yun } \\
\text { USA }\end{array}$ \\
\hline $\begin{array}{l}\text { Jonas Wensman } \\
\text { Sweden }\end{array}$ & $\begin{array}{l}\text { Kentaro Yamada } \\
\text { Japan }\end{array}$ & $\begin{array}{l}\text { Syed Sohail Zahoor Zaidi } \\
\text { Pakistan }\end{array}$ \\
\hline $\begin{array}{l}\text { Judith White } \\
\text { USA }\end{array}$ & $\begin{array}{l}\text { Seiya Yamayoshi } \\
\text { Japan }\end{array}$ & $\begin{array}{l}\text { Juan Zapata } \\
\text { USA }\end{array}$ \\
\hline $\begin{array}{l}\text { Gary Whittaker } \\
\text { USA }\end{array}$ & $\begin{array}{l}\text { Hanchun Yang } \\
\text { China }\end{array}$ & $\begin{array}{l}\text { Hassan Zaraket } \\
\text { Lebanon }\end{array}$ \\
\hline $\begin{array}{l}\text { Chris Wiethoff } \\
\text { USA }\end{array}$ & $\begin{array}{l}\text { Hwai-I Yang } \\
\text { Taiwan }\end{array}$ & $\begin{array}{l}\text { Mirjam Zeisel } \\
\text { France }\end{array}$ \\
\hline $\begin{array}{l}\text { Rebecca Wilkes } \\
\text { USA }\end{array}$ & $\begin{array}{l}\text { Kui Yang } \\
\text { USA }\end{array}$ & $\begin{array}{l}\text { Bo Zhang } \\
\text { China }\end{array}$ \\
\hline $\begin{array}{l}\text { David Williams } \\
\text { Australia }\end{array}$ & $\begin{array}{l}\text { Elizabeth Yanik } \\
\text { USA }\end{array}$ & $\begin{array}{l}\text { Dabing Zhang } \\
\text { China }\end{array}$ \\
\hline
\end{tabular}


Gui-Hong Zhang

China

Hengmu Zhang

China

Hong Zhang

USA

Jinyang Zhang

China

Ping Zhang

China
Jincun Zhao

USA

Jinzhuo Zhao

China

Weihao Zheng

China

Zhenyu Zhong

USA

Bin Zhou

USA
En-Min Zhou

China

Fengfeng Zhou

China

Guohui Zhou

China

Jiyong Zhou

China

Hua Zhu

USA 